\title{
sciendo
}

\section{Effects of Lower Extremity Muscle Fatigue on Knee Loading During a Forward Drop Jump to a Vertical Jump in Female Athletes}

\author{
by \\ Tzu Lin Wong', Chen Fu Huang², Po Chieh Chen ${ }^{2}$
}

The aim of this study was to examine changes in the kinematic and kinetic parameters of female athletes performing a forward drop jump to a vertical jump under muscle fatigue condition. Twelve female college athletes performed a forward drop jump to a vertical jump with and without muscle fatigue conditions. A motion capture system and two AMTI force plates were used to synchronously collect kinematic and kinetic data. Inverse dynamics were implemented to calculate the participant's joint loading, joint moment, and energy absorption. A paired sample ttest was used to compare statistical differences between pre-fatigue and post-fatigue conditions $(\alpha=.05)$. The forward trunk lean angle at initial foot contact, as well as the knee range of motion, total negative work and energy absorption contribution of the knee joint during the landing phase were significantly decreased under post-fatigue condition. The increased peak vertical ground reaction force and peak tibial anterior shear forces were also found under post-fatigue condition. These results indicated that muscle fatigue caused participants to change their original landing posture into stiff landing posture and decrease the energy absorption ability, which increased the tibial anterior shear forces. Therefore, female athletes should appropriately increase the knee flexion angle under muscle fatigue condition to reduce the risk of anterior cruciate ligament injuries.

Key words: energy absorption contribution, anterior cruciate ligament, joint moment.

\section{Introduction}

Landing movements with rapid deceleration and stoppages occur frequently in various sports (Coventry et al., 2006). Landing involves a combination of numerous biomechanical factors and is considered to be highly associated with anterior cruciate ligament (ACL) injuries (Lee et al., 2018; Krosshaug et al., 2007; Shimokochi et al., 2016), 70\% of which occur in noncontact situations (Boden et al., 2000). During the landing, muscles of the lower extremities absorb kinetic energy through eccentric contraction, which forces lower extremity joints to stretch and flex to absorb the impact force (Aizawa et al., 2016). Studies have reported that an ACL injury typically occurs within the first $100 \mathrm{~ms}$ after the foot contacts the ground during the landing (Koga et al., 2010; Krosshaug et al., 2007). This injury is primarily caused by anterior shear forces, specifically during knee valgus, knee varus, and internal rotation moments, increasing the load on the ACL considerably (Dai et al., 2014).

In order to prevent ACL injuries, studies have explored the potential landing biomechanical factors in ACL injuries during the landing. Some studies have incorporated changes in body posture to create different landing styles, such as self-selected, leaning forward, and upright landing (Blackburn and Padua, 2009; Shimokochi et al., 2013); some studies had an experimental

1 - Department of Physical Education, National Taipei University of Education, Taipei, Taiwan.

2 - Department of Physical Education, National Taiwan Normal University, Taipei, Taiwan. 
design involving changes in the foot landing position, such as toe-in and toe-out (Tran et al., 2016), or changes in trunk flexion angles (Blackburn and Padua, 2009). These studies have examined the relationship between ACL injuries and biomechanical variables such as lower extremity kinematics in the sagittal plane, vertical ground reaction force (GRF), anterior-posterior GRF, anterior tibial shear force, knee extension moment, and knee energy absorption contribution, thereby providing references for planning ACL injury prevention courses. Latent changes in lower extremity biomechanics observed when individuals perform the prefatigue landing have been indicated to effectively prevent ACL injuries. However, further clarification is required for the changes that occur in lower extremity biomechanics when individuals perform a landing under post-fatigue condition. Fatigue usually occurs in the later stages of sports matches or training sessions and increases the risk of noncontact ACL injuries (Chappell et al., 2005; Tamura et al., 2016). Researchers have also found that fatigue affects dynamic knee stability (Chappell et al., 2005; Coventry et al., 2006; Tamura et al., 2016). The dynamic control of the knee joint primarily depends on the eccentric shock attenuation capability of the muscles that surrounds it, especially the quadriceps muscle group. If eccentric capacity is lost because of fatigue, injury might result in damage to ligaments, cartilage, and bones (Chappell et al., 2005; Coventry et al., 2006). Studies have demonstrated that fatigue decreases knee power (Rodacki et al., 2001), jump height (Chappell et al., 2005), muscle work (Coventry et al., 2006), muscle contraction force (Rodacki et al., 2002), and physical coordination (Coventry et al., 2006; Zhang et al., 2000), in addition to delaying neuromuscular activation and expanding knee shear force and joint moment (Chappell et al., 2005). These biomechanical changes are believed to decrease shock absorption and knee stabilization during the landing. Accordingly, fatigue is strongly associated with ACL injuries. However, Zadpoor and Nikooyan (2012) concluded that no direct causal relationship exists between fatigue and leg injuries, which indicates that the effect of fatigue on the risk of leg injury requires further clarification.

Numerous studies have incorporated various landing positions into experiments to monitor ACL injury occurrence (Chinnasee et al., 2018), such as a one-foot or a two-foot drop landing (Heebner et al., 2017), counter movement jumps (Chinnasee et al., 2018), stop jumps (Heebner et al., 2017; Yu et al., 2006), a drop jump to a vertical jump (Ford et al., 2003), a forward drop jump to a vertical jump (Heebner et al., 2017), and a drop jump to a lateral vertical jump (Boo et al., 2018). When applying these landing positions, landing biomechanics must be used to evaluate rehabilitation effectiveness and ACL injury risks. The optimal landing position for evaluating ACL injury risks has yet to be determined. A study indicated that comparing these landing positions to obtain test results is difficult because each landing position differs in terms of biomechanical characteristics (Heebner et al., 2017). Cruz et al. (2013) evaluated the effects of a drop landing, a drop landing with a vertical jump and a forward drop jump with a vertical jump as landing positions for clinical ACL injury tests, revealing that the forward drop jump with a vertical jump contributed to a statistically significant increase in the abduction and flexion moments of the hip and knee compared with the other two landing positions. This confirmed that the three landing positions commonly applied for ACL injury tests differ in their kinematic and kinetic characteristics. Moreover, the forward drop jump landing with a vertical jump can be implemented for ACL injury evaluation in landing biomechanics and clinical studies.

The biomechanical influence of the landing has a greater effect on the legs of female athletes than of male athletes. Studies have indicated that female athletes exhibit larger knee flexion angles during initial ground contact, less knee angular displacement during the landing, greater vertical GRF and greater posterior GRF, greater anterior tibial shear force, and less total lower extremity energy absorption than male athletes during the landing (Briem et al., 2017). These have been reported as crucial factors influencing the occurrence of ACL injuries in female athletes. The purpose of this study was to explore the effect of post-fatigue the forward drop jump landing to a vertical jump on knee joint loads in female athletes, thereby providing a reference for ACL injury prevention. Based on previous findings, it was hypothesized that 
female athletes would exhibit greater kinematic and kinetic injury-predisposing factors such as a reduction in knee flexion, greater vertical GRF, greater posterior GRF, less knee total negative work, less knee energy absorption contribution and larger knee extension moments under the post-fatigue condition compared with the prefatigue condition.

\section{Methods}

\section{Participants}

Twelve female collage athletes (age: 21.33 \pm 1.49 yrs, body height: $1.64 \pm 0.05 \mathrm{~m}$, body mass: $59.88 \pm 6.18 \mathrm{~kg}$ ) with no history of cardiovascular, respiratory, neurological, lower extremity injury and ACL injury volunteered for this study. Participants were asked to list their primary sport; eight reported basketball and four reported volleyball. All participants had at least five years of experience in their reported sport. They had trained four to six times per week and previously engaged in these sports at the college level. The study was approved by the National Taiwan Normal university ethics committee and participants' informed consent was provided prior to their participation (participant under 20 years old required parental consent).

\section{Experimental Procedure}

A motion capture system consisted of four high-speed cameras (sampling rate: $120 \mathrm{~Hz}$, model: Mega Speed MS30k, Minnedosa, MB, Canada) and two force plates (sampling rate: 1200 Hz, model: AMTI BP600900, Advanced Medical Technology, Inc., Watertown, MA, USA) which were synchronized to obtain kinematic and kinetic data. Forty-five retro-reflective markers (14-mm diameter) were attached bilaterally to each of the following locations to track the motion of the body segments. Marker locations included the head (front of the head, back of the head), torso (seventh cervical vertebrae, $10^{\text {th }}$ thoracic vertebrae, clavicle, sternum, and one tracking marker), arms (acromio-clavicular joint, lateral/medial epicondyle, styloid processes, second metacarpal, and tracking markers for each upper arms and forearms), pelvis (ASIS and PSIS), legs (greater trochanter, lateral/medial epicondyle of knee, lateral/medial malleolus, and tracking markers for each thigh and shank), and feet (second metatarsal heads, fifth metatarsal heads, and calcaneus). Prior to data collection, cameras were calibrated according to the manufacturer's recommendation using a Direct Linear Transformation algorithm with a 32-point, customized three-dimensional coordinate frame (length: $100 \mathrm{~cm}$, width: $100 \mathrm{~cm}$, and height: 200 $\mathrm{cm})$. A static trial was captured with each participant standing still, with arms across the chest, to align the joint coordinates to the laboratory recording instruments.

Each participant started with a five-min warm up (three-minute running with a speed of 3 $\mathrm{m} / \mathrm{s}$ and two-minute stretching) and then performed three maximal vertical double-legged jumps to determine their maximal vertical jump height. The participant was allowed to practice and familiarize the forward drop jump to a vertical jump skill before data collection. After a five-min rest period, the participant was asked to perform the forward drop jump to a vertical jump for pre-fatigue data collection. A $30 \mathrm{~cm}$ box was placed at a distance equal to $50 \%$ of the participant's height from the front edge of the force plates. The participant stood on the top of the 30-cm-high box and performed a forward jump onto two force plates with both feet at the same time and subsequently performed a vertical maximum jump (Figure 1) (Padua et al., 2009; Tran et al., 2016). After five successful trials, participants performed sets of a fatigue protocol designed to induce fatigue until a $10 \%$ decrement in their maximal vertical jump height was reached. The fatigue protocol consisted of 50 stepups onto a $30-\mathrm{cm}$ box, followed by 15 maximaleffort single-legged vertical jumps (Liederbach et al., 2014). At the end of each set, participant's maximum vertical jump height was assessed, and participants were asked to rate their perceived exertion using the Borg CR-10 scale (Borg et al., 2010). When participants attained a $10 \%$ decrement in vertical jump height and the Borg CR-10 scale level 17, they performed the forward drop jump to a vertical jump again for postfatigue data collection. The average of five successful trials was reported. A trial with the entire foot contacting the force plates was considered successful.

\section{Data analysis}

Kinematic and kinetic data were analysed using Kwon 3D motion-analysis software (Visol, Gwangmyeong, South Korea). Raw kinematic data were low-pass filtered using a fourth-order, 
zero-phase-lag Butterworth filter with a cut-off frequency of $10 \mathrm{~Hz}$ (Decker et al., 2003), time synchronized with the kinetic data, and resampled at $1200 \mathrm{~Hz}$. Kinetic data were low-pass filtered at $60 \mathrm{~Hz}$ with a fourth-order, zero-phaselag Butterworth filter (Norcross et al., 2013; Schmitz et al., 2007; Shultz et al., 2009) and combined with kinematic and anthropometric data to calculate the net joint moments at the hip and the ankle and the net internal force on the shank at the knee joint using an inverse dynamics solution.

Anatomical reference frames for the body segments were defined for the $\mathrm{x}$-axis as positive anteriorly, the $y$-axis as positive medially, and the $\mathrm{z}$-axis as positive superiorly. Hip and knee angles were calculated using Euler angle definitions with a rotational sequence of $z, y^{\prime}, x^{\prime \prime}$. Trunk angles were calculated as the trunk reference frame relative to the thigh reference frame. Hip angles were calculated as the sacrum reference frame relative to the thigh reference frame, while knee angles were calculated as the shank reference frame relative to the thigh reference frame. The body mass of each participant was recorded before data collection and was used to normalize the dependent kinetic variables.

The study used custom computer software to multiply sagittal plane joint angular velocities $(\omega)$ and net joint moments $(\mathrm{M})$ to generate knee joint power curves $(\mathrm{P})$ for each landing trial $(\mathrm{P}-\mathrm{A} \omega$ ). The negative portion of the joint power curves (i.e., when net joint moment and joint angular velocity were in opposite directions and indicated eccentric muscle action) were integrated to calculate negative mechanical joint work during the landing phase (from the initial ground contact to the minimal vertical position of the whole-body center of mass (CM)) (Decker et al., 2003). Finally, percentage energy absorption contribution (EAC) of the knee joint to the total negative limb energy was calculated as followed (Garrison, 2018):

$$
E A C_{\text {knee }}^{\text {negative }}=\left(W_{\text {knee }}^{\text {negative }} / W_{\text {Limb }}^{\text {negative }}\right) \times 100
$$

All dependent variables were averaged across the 5 forward drop jump to a vertical jump trials of each participant before statistical analysis.

\section{Statistical Analysis}

A paired sample $t$-test was used to evaluate whether kinematics and kinetics were significant in sagittal plane demands between pre-fatigue and post-fatigue conditions. All statistical analyses were conducted in SPSS (version 21; SPSS Inc., Chicago, IL, USA), and the alpha level was set up at .05. Cohen's effects sizes were calculated for variables to compare between the two conditions.

\section{Results}

As shown in Table 1, the knee flexion at initial ground contact significantly increased, but the trunk forward lean angle decreased under post-fatigue condition $(p<.05)$. The ROM at knee joint was also decreased by $9.8 \%$ during the landing phase under post-fatigue condition $(p<$ $.05)$.

With regard to kinetics (Table 2), a significant increase was observed in the peak vertical GRF and anterior tibial shear force under muscle fatigue condition $(p<.05)$. However, no significant changes were observed in knee extension moment $(p>.05)$. The total negative work and energy absorption on the knee joint decreased significantly $(p<.05)$, but no significant changes were observed in the total positive work on the knee $(p>.05)$ under muscle fatigue condition.

\section{Discussion}

Under muscle fatigue condition, the participants' knee ROM and trunk flexion angle decreased significantly, indicating that during muscle fatigue, the human body adopts a relatively stiff landing position, causing a decrease in the peak knee and trunk flexion time during a drop landing. A previous study indicated that decreased knee ROM might increase knee ligamentous stress by decreasing the strength of the hamstrings to resist anterior shear forces on the tibia (Chappell et al., 2005). Decreased maximum knee flexion joint angles became a compensation strategy to prevent collapse of the CM because of fatigue in the quadriceps muscle group. In the compensation mechanism, a minimal smaller knee joint extension moment under fatigue condition was observed as a strategy to reinforce the lower extremity stability and prevent subsequent collapse of the CM. The decreased knee extension joint moments by increasing joint stiffness, further supported participants to perform faster 
concentric contractions toward a jump (Rodacki et al., 2001). As shown in Table 1, the knee flexion angle increased at initial ground contact, but the knee ROM during the landing phase decreased significantly under muscle fatigue condition. These results are consistent with a previous study, which showed that fatigue resulted in greater knee flexion angles at initial ground contact and less excursion during the landing (Briem et al., 2017). Blackburn and Padua (2009) posited that a larger trunk flexion angle during landing caused larger hip and knee flexion angles; moreover, a landing with the trunk flexion effectively reduced vertical GRF and quadriceps myoelectric amplitude. Therefore, the trunk flexion is necessary to prevent ACL injury during the landing. However, the participants' trunk flexion angles in this study decreased significantly under muscle fatigue condition; accordingly, the participants' trunks might have adopted stiff landing positions under muscle fatigue condition, raising their risks of sustaining ACL injuries.

Table 1

Kinematic variables of the pre-fatigue and post-fatigue conditions $(N=12)$

\begin{tabular}{lccccc}
\hline \multicolumn{1}{c}{ Variable } & Pre-fatigue & Post-fatigue & $t$ & $p$ & Cohen's $d$ \\
\hline At initial ground contact & & & & & \\
Knee flexion angles $\left(^{\circ}\right)$ & $22.33 \pm 6.65$ & $26.07 \pm 3.41$ & $-2.884^{*}$ & .018 & .69 \\
Hip flexion angles $\left(^{\circ}\right)$ & $22.80 \pm 5.65$ & $24.79 \pm 7.21$ & 1.258 & .240 & .31 \\
$\quad$ Trunk forward lean angle $\left(^{\circ}\right)$ & $90.62 \pm 3.63$ & $86.04 \pm 4.42$ & $-5.191^{*}$ & .001 & 1.13 \\
During landing phase & & & & & .65 \\
$\quad$ Knee ROM $\left(^{\circ}\right)$ & $83.28 \pm 13.5$ & $75.12 \pm 11.2$ & $-3.278^{*}$ & .010 & .38 \\
$\quad$ Hip ROM $\left(^{\circ}\right)$ & $69.54 \pm 16.9$ & $63.93 \pm 12.1$ & -1.580 & .148 & .65 \\
\hline
\end{tabular}

Values are mean $\pm S D,{ }^{*}$ Denotes significant difference at $p<.05$; Abbreviation: ROM, range of motion

Table 2

Kinetic variables of the pre-fatigue and post-fatigue conditions $(n=12)$

\begin{tabular}{lccccc}
\hline \multicolumn{1}{c}{ Variable } & Pre-fatigue & Post-fatigue & $t$ & $p$ & Cohen's $d$ \\
\hline Peak posterior GRF (BW) & $0.54 \pm 0.07$ & $0.59 \pm 0.09$ & 2.21 & .055 \\
Peak vertical GRF (BW) & $2.98 \pm 0.34$ & $3.11 \pm 0.37$ & $3.55^{*}$ & .006 & .06 \\
Anterior tibial shear force (BW) & $0.48 \pm 0.09$ & $0.53 \pm 0.06$ & $2.79^{*}$ & .021 \\
Knee extension moment & $2.88 \pm .52$ & $3.11 \pm .88$ & 1.41 & .191 \\
$\left(\mathrm{Nm} \cdot \mathrm{kg}^{-1}\right.$ ) & & & .65 \\
Total positive work on knee $\left(\mathrm{J} \cdot \mathrm{kg}^{-1}\right)$ & $242.82 \pm 105.69$ & $213.26 \pm 95.55$ & -1.32 & .219 \\
Total negative work on knee $\left(\mathrm{J} \cdot \mathrm{kg}^{-1}\right)$ & $300.82 \pm 82.17$ & $237.51 \pm 80.13$ & $-2.77^{*}$ & .022 \\
Knee energy absorption $(\%)$ & $42.36 \pm 16.28$ & $31.25 \pm 14.3$ & $-2.33^{*}$ & .018 & .78 \\
\hline
\end{tabular}

Values are mean $\pm S D$; ${ }^{*}$ Denotes significant difference at $p<.05$;

Abbreviation: GRF, ground reaction force 


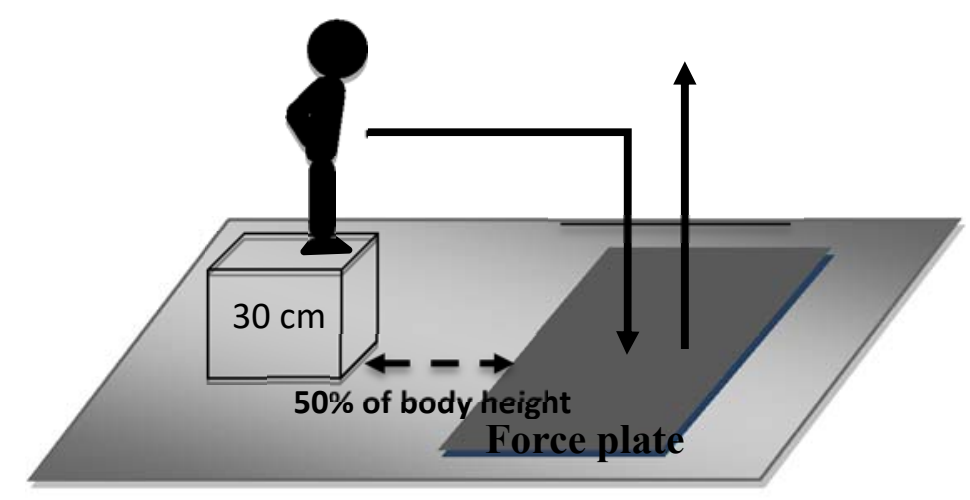

Figure 1

Schematic diagrams of the forward drop jump to a vertical jump task

Higher peak vertical GRF was also found to be associated with lower knee flexion angles under muscle fatigue condition, and this finding was supported by a study of Hewett et al. (2013). The effect of GRF has been a prominent topic in landing biomechanics. GRFs could be influenced by muscle contraction, muscle fatigue, body motion, and body posture (James et al., 2007). During the landing, an increase in peak GRF must be counterbalanced through an increase in knee extension moment, and the knee extension moment generated by the quadriceps leads to an increased proximal anterior shear force, thereby heightening the risk of ACL injuries (Chappell et al., 2005). According to Shimokochi et al. (2016), when an individual lands on the sagittal plane, vertical GRF is significantly correlated with peak tibial axial force and anterior tibial shear force $(\mathrm{r}=$ .99 and .76, respectively). Therefore, muscle fatigue affects the anterior tibial shear force generated during the landing. In the present study, significant increases were observed in the peak vertical GRF and anterior tibial shear force under muscle fatigue condition.

Studies have reported a high correlation between ACL injury and the knee flexion angle during the landing (Boden et al., 2009; Koga et al., 2010; Shimokochi et al., 2016). A stiff landing has been indicated to cause less joint angular displacement than a soft landing, which leads to a larger GRF against the lower extremities. Moreover, GRF is positively associated with knee extension moment (Tran et al., 2016). Koga et al. (2010) reported that tibial external rotation, knee valgus, and knee range of motion were commonly used as indicators of noncontact ACL injuries during the landing. Because of knee compression force and the recruitment order of quadriceps motor units, anterior tibial shear force is reduced through ground-impact energy absorption and knee range of motion during the landing. Therefore, changes in landing postures in the sagittal plane are associated with a risk of ACL injury (Shimokochi et al., 2016).

ACL injuries incurred during a landing are caused by an increase in ACL tension, which is primarily induced by the anterior shear force at the ends of the tibia (Shimokochi et al., 2016). This study employed inverse dynamic equations to calculate the force exerted on human joints. In particular, anterior tibial shear force was defined as the load vertical to the major axis of bones. The 
angular movement in human limbs is caused by the combined forces of muscle contractions. Therefore, net muscle joint moment can be used as an indicator for the combined forces of the quadriceps on the knee and posterior compartment of the leg (Yu et al., 2006) during movement. During the landing, anterior tibial shear force is generated on the knee by body weight, causing the knee to bend. Subsequently, the force of gravity causes knee extension moment to increase, leading the quadriceps to increase its contraction force to counterbalance the moment. The greater the muscle force generated in the quadriceps, the higher the tension in the patellar tendon. Therefore, stronger shear and axial forces in the joints lead to higher risks of injuries. This study discovered that the hip moment and anterior tibial shear force generated in the forward drop jump to a vertical jump after muscle fatigue were greater than those before muscle fatigue; a significant difference was also observed between the two conditions. Studies on stop jumps have indicated that the maximum net joint moment is substantially correlated with proximal tibia anterior shear force, and that anterior tibial shear force increases along with fatigue (Chappell et al., 2005; Yu et al., 2006). Accordingly, the anterior tibial shear force in female athletes increases during fatigue, increasing their risks of sustaining ACL injuries.

During the landing, plantar flexion moment in the hips, knees, and ankle joints is generated through eccentric muscle contractions, through which the joints' range of motion is controlled and kinetic energy in the human body is absorbed. Energetic analysis can be conducted to examine the neuromuscular control strategies of joint extensors when the leg absorbs impact energy after the landing, thereby providing further information on the risk of ACL injuries. Studies have indicated that reducing knee flexion angles may lower knee power and, in turn, knee energy absorption (Boo et al., 2018; Coventry et al., 2006). Furthermore, stiff landing positions reduce joint energy absorption and increase the loading of ACL (Decker et al., 2003). Therefore, variables such as knee negative work and the contribution of the knee to total negative work considerably influence ACL loads during the landing (Lee et al., 2018).

Studies on landing movements have reported significant differences in lower extremity biomechanics between male and female athletes; female athletes were found to be at a higher risk of sustaining ACL injuries than male athletes (Borotikar et al., 2008). Moreover, this study adopted the forward drop jump to a vertical jump, which increases athletes' knee and trunk flexion angles as well as knee flexion moments more easily than other landing positions adopted by relevant studies to evaluate the risks related to ACL injuries. From the perspective of kinematics and kinetics, the forward drop jump to a vertical jump requires more energy than other landing positions to enable athletes' bodies to absorb strong impact forces upon movement completion. Fatigue weakens the neuromuscular control capacity of human bodies and increases the difficulty in movement control after the landing, thus heightening the risks of ACL injuries.

This study indicates that fatigue instantly affects the postures and sagittal-plane biomechanical variables of female athletes performing the forward drop jump to a vertical jump. Fatigue considerably increases the vertical GRF and anterior tibial shear force and reduces knee and trunk flexion angles as well as knee energy absorption during the landing. Accordingly, muscle fatigue is strongly associated with lower extremity and ACL injury occurrence, particularly in female athletes.

\section{References}

Aizawa J, Ohji S, Koga H, Masuda T, Yagishita K. Correlations between sagittal plane kinematics and landing impact force during single-leg lateral jump-landings. J Phys Ther Sci, 2016; 28(8): 2316-2321

Blackburn JT, Padua DA. Sagittal-plane trunk position, landing forces, and quadriceps electromyographic activity. J Athl Train, 2009; 44(2): 174- 179

Boden BP, Dean GS, Feagin JA, Garrett WE. Mechanisms of anterior cruciate ligament injury. Orthopedics, 2000; 23(6): 573-578

Boden P, Torg JS, Knowles SB, Hewett TE. Video analysis of anterior cruciate ligament injury: Abnormalities in hip and ankle kinematics. Am J Sports Med, 2009; 37(2): 252-259 
Boo ME, Garrison JC, Hannon JP, Creed KM, Goto S, Grondin AN, Bothwell JM. Energy absorption contribution and strength in female athletes at return to sport after anterior cruciate ligament reconstruction: comparison with healthy controls. Orthop J Sports Med, 2018; 6(3): 2325967118759522

Borg E, Borg G, Larsson K, Letzter M, Sundblad BM. An index for breathlessness and leg fatigue. Scand J Med Sci Sports, 2010; 20(4): 644-650

Borotikar BS, Newcomer R, Koppes R, McLean SG. Combined effects of fatigue and decision making on female lower limb landing postures: Central and peripheral contributions to ACL injury risk. Clin Biomech, 2008; 23(1): 81-92

Briem K, Jonsdottir KV, Arnason A, Sveinsson P. Effects of sex and fatigue on biomechanical measures during the drop-jump task in children. Orthop J Sports Med, 2017; 5(1): 2325967116679640

Chappell JD, Herman DC, Knight BS, Kirkendall DT, Garrett WE, Yu B. Effect of fatigue on knee kinetics and kinematics in stop-jump tasks. Am J Sports Med, 2005; 33(7): 1022-1029

Chinnasee C, Weir G, Sasimontonkul S, Alderson J, Donnelly C. A biomechanical comparison of single-leg landing and unplanned sidestepping. Int J Sports Med, 2018; 39(8): 636-645

Coventry E, O'Connor, KM, Hart BA, Earl JE, Ebersole, KT. The effect of lower extremity fatigue on shock attenuation during single-leg landing. Clin Biomech, 2006; 21(10): 1090-1097

Cruz A, Bell D, McGrath M, Blackburn T, Padua D, Herman D. The effects of three jump landing tasks on kinetic and kinematic measures: implications for ACL injury research. Res Sports Med, 2013; 21(4): 330342

Dai B, Mao D, Garrett WE, Yu B. Anterior cruciate ligament injuries in soccer: Loading mechanisms, risk factors, and prevention programs. J Sport and Health Sci, 2014; 3(4): 299-306

Decker MJ, Torry MR, Wyland DJ, Sterett WI, Steadman JR. Gender differences in lower extremity kinematics, kinetics and energy absorption during landing. Clin Biomech, 2003; 18(7): 662-669

Ford KR, Myer GD, Hewett TE. Valgus knee motion during landing in high school female and male basketball players. Med Sci Sports Exerc, 2003; 35(10): 1745-1750

Heebner NR, Rafferty DM, Wohleber MF, Simonson AJ, Andrew-Reinert ML, Sell TC. Landing kinematics and kinetics at the knee during different landing tasks. J Athl Train, 2017; 52(12): 1101-1108

Hewett TE, DiStasi SL, Myer GD. Current concepts for injury prevention in athletes after anterior cruciate ligament reconstruction. Am J Sports Med, 2013; 41(1): 216-224

James CR, Herman JA, Dufek JS, Bates BT. Number of Trials Necessary to Achieve Performance Stability of Selected Ground Reaction Force Variables During Landing. J Sports Sci Med, 2007; 6(1): 126-134

Koga H, Nakamae, A, Shima Y, Iwasa J, Myklebust G, Engebretsen L, Bahr R, Krosshaug T. Mechanisms for noncontact anterior cruciate ligament injuries: knee joint kinematics in 10 injury situations from female team handball and basketball. Am J Sports Med, 2010; 38(11): 2218-2225

Krosshaug T, Nakamae A, Boden BP, Engebretsen L, Smith G, Slauterbeck JR, Bahr R. Mechanisms of anterior cruciate ligament injury in basketball. Am J Sports Med, 2007; 35(3): 359-367

Lee J, Song Y, Shin CS. Effect of the sagittal ankle angle at initial contact on energy dissipation in the lower extremity joints during a single-leg landing. Gait Posture, 2018; 62: 99-104

Liederbach M, Kremenic IJ, Orishimo KF, Pappas E, Hagins M. Comparison of landing biomechanics between male and female dancers and athletes, part 2: Influence of fatigue and implications for anterior cruciate ligament injury. Am J Sports Med, 2014; 42(5): 1089-1095

Norcross MF, Lewek MD, Padua DA, Shultz SJ, Weinhold PS, \& Blackburn JT. Lower extremity energy absorption and biomechanics during landing, part I: sagittal-plane energy absorption analyses. J Athl Train, 2013; 48(6): 748-756

Padua DA, Marshall SW, Boling MC, Thigpen CA, Garrett WE, Jr, Beutler AI. The landing error scoring system (less) is a valid and reliable clinical assessment tool of jump-landing biomechanics: the JUMPACL study. Am J Sports Med, 2009; 37: 1996-2002

Rodacki AL, Fowler NE, Bennett SJ. Multi-segment coordination: Fatigue effects. Med Sci Sports Exerc, 2001; 33: $1157-1167$

Schmitz RJ, Kulas AS, Perrin DH, Riemann BL, Shultz SJ. Sex differences in lower extremity biomechanics during single leg landings. Clin Biomech, 2007; 22: 681-688 
Shimokochi Y, Ambegaonkar JP, Meyer EG. Changing sagittal-plane landing styles to modulate impact and tibiofemoral force magnitude and directions relative to the tibia. J Athl Train, 2016; 51(9): 669-681

Shimokochi Y, Meyer E, Lee SY, Ambegaonkar JP, Shultz SJ. Changing sagittal plane body positions influences noncontact anterior cruciate ligament injury risk during single-leg landings. Knee Surg Sports Traumatol Arthrosc, 2013; 21(4): 888-897

Shultz SJ, Nguyen A-D, Leonard MD, and Schmitz RJ. Thigh strength and activation as predictors of knee biomechanics during a drop jump task. Med Sci Sports Exerc, 2009; 41(4): 857-866

Tamura A, Akasaka, K, Otsudo T, Sawada Y, Okubo Y, Shiozawa J. Fatigue alters landing shock attenuation during a single-leg vertical drop jump. Orthop J Sports Med, 2016; 4(1): 2325967115626412

Tran AA, Gatewood C, Harris AHS, Thompson JA, Dragoo J. The effect of foot landing position on biomechanical risk factors associated with anterior cruciate ligament injury. J Exp Orthop, 2016; 3(13): $1-7$

Yu B, Lin CF, Garrett WE. Lower extremity biomechanics during the landing of a stop-jump task. Clin Biomech, 2006; 21(3): 297-305

Zadpoor AA, Nikooyan AA. The effects of lower-extremity muscle fatigue on the vertical ground reaction force: A meta-analysis. J Eng Med, 2012; 226(8): 579-588

Zhang SN, Bates BT, Dufek JS. Contributions of lower extremity joints to energy dissipation during landings. Med Sci Sports Exerc, 2000; 32(4): 812-819

\section{Corresponding author:}

\section{Po Chieh Chen}

Department of Physical Education, National Taiwan Normal University

No. 88, Sec. 4, TingJhou Rd., WunShan Distinct, Taipei City 11677, Taiwan.

Email: chenpojay@gmail.com

Phone: +886-2-29377199 \#113

Fax: +886-2-29377315 